\title{
Development of New-Onset Cervical Deformity in Nonoperative Adult Spinal Deformity Patients With 2-Year Follow-Up
}

PETER G. PASSIAS, MD,${ }^{1}$ CYRUS M. JALAI, BA, ${ }^{1}$ NANCY WORLEY, MS,,${ }^{1}$ SHALEEN VIRA, MD,${ }^{1}$ JUSTIN K. SCHEER, BS, ${ }^{2}$ JUSTIN S. SMITH, MD,${ }^{3}$ SUBARAMAN RAMACHANDRAN, MD,${ }^{1}$ ALEXANDRA SOROCEANU, MD,${ }^{4}$ SAMANTHA R. HORN, BA, ${ }^{1}$ GREGORY W. POORMAN, BA, ${ }^{1}$ THEMISTOCLES S. PROTOPSALTIS, MD ${ }^{1}$ ERIC O. KLINEBERG, $\mathrm{MD},{ }^{5}$ DANIEL M. SCIUBBA, MD, ${ }^{6}$ HAN JO KIM, MD, ${ }^{7}$ D. KOJO HAMILTON, MD ${ }^{8}$ RENAUD LAFAGE, $\mathrm{MS},{ }^{7}$ VIRGINIE LAFAGE, PHD ${ }^{7}$ CHRISTOPHER P. AMES, $\mathrm{MD}^{9}$; INTERNATIONAL SPINE STUDY GROUP (ISSG)

${ }^{1}$ Department of Orthopaedic Surgery, New York University Hospital for Joint Diseases, New York, New York, ${ }^{2}$ School of Medicine, University of California, San Diego, La Jolla, California, ${ }^{3}$ Department of Neurosurgery, University of Virginia Medical Center, Charlottesville, Virginia, ${ }^{4}$ Orthopaedic Surgery, University of Calgary, Calgary, Alberta, Canada, ${ }^{5}$ Department of Orthopaedic Surgery, University of California, Davis, Sacramento, California, ${ }^{6}$ Department of Neurosurgery, The Johns Hopkins University, Baltimore, Maryland, ${ }^{7}$ Department of Orthopaedic Surgery, Hospital for Special Surgery, New York, New York, ${ }^{8}$ Department of Neurological Surgery, University of Pittsburgh School of Medicine, Pittsburgh, Pennsylvania, ${ }^{9}$ Department of Neurological Surgery, University of California, San Francisco, San Francisco, California

\begin{abstract}
Purpose: Evaluate the presence of new-onset cervical deformity $(\mathrm{CD})$ in nonoperative adult spinal deformity (ASD) patients with extended follow-up, with consideration for predictors, prevalence, and impact on patient-reported outcomes.

Methods: Retrospective review of a prospective nonoperative ASD cohort. New onset CD patients at 1- (CD-1Y) and 2-year (CD-2Y) follow-up were defined as displaying baseline cervical alignment. Univariate analyses determined differences in radiographic parameters and outcome scores of $\mathrm{CD}$ and maintained-cervical-alignment patients. Multivariate binary logistic regression models determined new-onset CD predictors.

Results: A total of 143 patients were included (mean age 54 years, mean body mass index $25.6 \mathrm{~kg} / \mathrm{m}^{2}, 86 \%$ female). Cervical deformity rate was $38.5 \%$ at baseline. New-onset CD incidence at 1 - and 2-year follow-up was $30.0 \%$ and $41.7 \%$, respectively. Global sagittal profile comparison of CD-1Y/CD-2Y versus maintained cervical alignment cases revealed no differences $(P>.05)$ at any interval. Baseline C2-C7 sagittal vertical axis (SVA) was associated with increased new-onset $\mathrm{CD}$ risk at 1 (odds ratio [OR] 1.14, $P=.025$ ) and 2 years (OR 1.04, $P=.032$ ); prior spine surgical history was associated with $\mathrm{CD}$ risk at 1-year follow-up (OR 6.75, $P=.047)$; baseline $\mathrm{C} 2$ slope was associated with increased CD risk at 2-year follow-up (OR 1.12, $P=.041)$. CD development did not significantly impact health-related quality of life $(P>.05)$.

Conclusions: Cervical deformity can manifest in nonoperative ASD patients: $30.0 \%$ at 1-year follow-up, and $41.7 \%$ at 2-year follow-up. Progressive CD manifested independently of thoracolumbar profile changes. Increased baseline C2-C7 SVA, C2 slope, and prior surgical history increased new-onset CD odds at 1 and 2 years.
\end{abstract}

Lumbar Spine

Keywords: cervical deformity, adult spinal deformity, new-onset cervical deformity, nonoperative

\section{INTRODUCTION}

Successful efforts in addressing adult spinal deformity (ASD) regarding the sagittal plane have been recently undertaken, and have also begun to incorporate analyses of upper spinal regions for the maintenance of global alignment. ${ }^{1,2}$ While surgical intervention for ASD has demonstrated effectiveness and is regarded as the more viable treatment approach in select patients, conservative manage- ment remains the mainstay initial treatment form ${ }^{3}$; however, the lack of corrective alignment with the potential for subsequent degeneration during nonoperative care may portend to clinically important compensatory changes in the cervical spine. Cervical changes in ASD patients recommended for conservative treatment have not been investigated, and this gap in knowledge informs the present study. 
Radiographic analyses of cervical alignment (CA) are marked by variations in defining "cervical deformity" (CD) in contrast to CA. Past efforts have highlighted a primary marker of $\mathrm{CD}$ as a large C2-C7 sagittal vertical axis (SVA, usually $\geq 4 \mathrm{~cm}$ ), which has been correlated with poor clinical outcomes. ${ }^{4}$ Building on this, Scheer et al in 2013 utilized the chin-brow vertical angle and C2-SVA to characterize $\mathrm{CD}$, citing horizontal gaze maintenance and neck pain reduction as primary deformity drivers. ${ }^{5,6}$ As the literature has evolved, a more comprehensive definition of CD has been postulated by Passias et al to incorporate 3 cervical parameters: $\mathrm{T} 1$ slope minus cervical lordosis $>20^{\circ}, \mathrm{C} 2-\mathrm{C} 7$ kyphosis $\geq 10^{\circ}$, and $\mathrm{C} 2-\mathrm{C} 7 \mathrm{SVA} \geq 4 \mathrm{~cm}^{7}$ Conversely, few efforts however have been directed toward determining radiographic ranges of "normative" CA. Nonetheless, the relation between cervical and thoracolumbar deformities is increasingly investigated, though chiefly following surgical deformity correction. Oh et al recently reported baseline cervical hyperlordosis deformity $\left(>15^{\circ}\right)$ rates in $48.9 \%$ of ASD patients, which persisted at 3 months and 2 years after thoracolumbar surgical correction. ${ }^{8}$ These authors also observed that $\mathrm{C} 2-\mathrm{C} 7$ SVA increased following thoracolumbar surgery $\left(41.7^{\circ}\right.$ to $\left.47.0^{\circ}\right){ }^{8}$ Postoperative CD after ASD surgery as reported by Passias et al was similarly high at $47.7 \%{ }^{7}$

As the cervical spine is the most mobile segment of the spinal column, it is susceptible to developing changes in alignment in order to maintain the head over the pelvis, thereby facilitating horizontal gaze. Moreover, CA pathology warrants specific consideration, with increasing reports of correlations between health-related quality of life (HRQoL), adverse health and function effects on patients, and sagittal spinal alignment parameters. ${ }^{9-12}$ Thoracolumbar deformities thus have important ramifications on upper and lower segmental alignment. However, reciprocal changes in neighboring spine segments have not been thoroughly quantified in nonoperative patients. Effectively, present understanding of the mechanisms by which such changes occur in both operative and nonoperative populations, and whether they are inherent to the disease itself or the intervention, remains thus incomplete. As such, this analysis endeavored to report on newonset CD among patients with preexisting and maintained thoracolumbar spinal deformities treated nonoperatively.

\section{METHODS}

\section{Data Source}

This study is a retrospective analysis of a prospectively collected multi-center database for consecutively enrolled operative and nonoperative ASD patients at 11 participating centers around the United States from 2008 to 2014. Prior to study initiation, each participating site received Institutional Review Board approval. Inclusion criteria for the database was patient age $>18$ years with a diagnosis of ASD, defined as the presence of: scoliosis $\geq 20^{\circ}$ (measured by major coronal Cobb angle), SVA (distance between the C7 plumb line and posterior superior margin of the sacrum) $\geq 5 \mathrm{~cm}$, pelvic tilt $\geq 25^{\circ}$, or thoracic kyphosis $>60^{\circ}$. The decision to pursue operative or nonoperative treatment for each patient was arrived at during consultation between the patient and surgeon, and was ultimately guided by patient choice. There was no attempt to randomize patients in this study. This study included only ASD patients treated nonoperatively with complete demographic, radiographic, and HRQoL data at baseline and 1and 2-year follow-up examinations.

\section{Assessment of CD}

$\mathrm{CD}$ has been previously radiographically defined as $\mathrm{T} 1$ slope minus cervical lordosis (T1S - CL) $>20^{\circ}, \mathrm{C} 2-\mathrm{C} 7 \mathrm{SVA} \geq 40 \mathrm{~mm}$, or C2-C7 kyphosis $(\mathrm{C} 2-\mathrm{C} 7 \mathrm{CK})<-10^{\circ}$. ${ }^{7}, 11$ Patients included within our $\mathrm{CD}$ cohort met $\geq 2$ of the aforementioned criteria on baseline or yearly follow-up radiographs. Cervical alignment was defined as $\geq 2$ of the following parameters on baseline and yearly follow-up radiographs: $\mathrm{T} 1 \mathrm{~S}-\mathrm{CL}<20^{\circ}, \mathrm{C} 2-\mathrm{C} 7$ $\mathrm{SVA} \leq 40 \mathrm{~mm}$, or $\mathrm{C} 2-\mathrm{C} 7 \mathrm{CK} \leq 0^{\circ}$. Nonoperative ASD patients were classified as displaying "newonset CD" at each follow-up time point and used for analysis only if they were categorized as displaying CA on their baseline radiographs.

\section{Data Collection and Analysis}

Collected data included patient demographics (age, gender, body mass index, medical comorbidities) and HRQoL outcome scores at each follow-up interval, including the following: Oswestry Disability Index (ODI), Short-Form (SF-36) Health Survey Mental and Physical component summaries, Scoliosis Research Society (SRS) patient questionnaire 


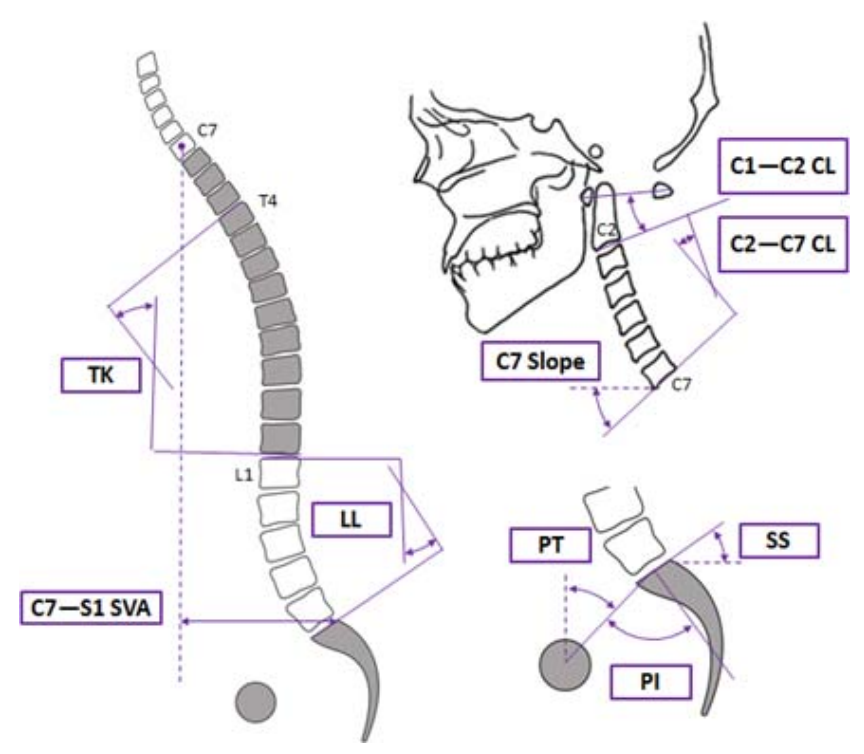

Figure 1. Spinopelvic parameters: LL indicates lumbar lordosis; TK, thoracic kyphosis; PI, pelvic incidence; SS, sacral slope; PT, pelvic tilt; SVA, C7-S1 sagittal vertical axis; and CL, C1-C2 and C2-C7 cervical lordosis. The parameter of $\mathrm{C} 7$ slope is also depicted.

(SRS-22r) activity), pain, appearance, satisfaction, mental, and total.

Regional and global radiographic parameters (Figure 1) were analyzed and measured from fulllength free-standing lateral spine radiographs (91-4 $\mathrm{cm}$ [36-inch] cassette) with visible cervical spine at baseline, 1-year follow-up, and 2-year follow-up with a validated software system (SpineView, ENSAM PariTech, Paris, France) at a single center with high accuracy and reliability. ${ }^{13-15}$ Cervical spine measurements included the following: C2-C7 cervical lordosis (angle between lower endplates of $\mathrm{C} 2$ and C7); C2 and C7 slopes (angle between horizontal and lower vertebral endplate); SVA (distance between the $\mathrm{C} 7$ plumb line and posterior superior margin of the sacrum) for $\mathrm{C} 2-\mathrm{C} 7$ and $\mathrm{C} 2$ T3; mismatch between T1-slope and cervical lordosis (T1S-CL), the cervical analog of pelvic incidence minus lumbar lordosis (PI - LL). The following pelvic parameters were obtained: pelvic tilt, pelvic incidence, and sacral slope. Collected thoracolumbar measurements included coronal Cobb angles of thoracic and lumbar curves, T (T4T12; Cobb angle between superior T4 endplate and inferior T12 endplate), lumbar lordosis (Cobb angle between superior endplates of L1 and S1), SVA (C7 plumb line relative to $\mathrm{S} 1$ ), global angulation (C2$\mathrm{S} 1$ ), and T1 pelvic angle (angle between femoral head axis to $\mathrm{T} 1$ center, and line from femoral head axis to middle of $\mathrm{S} 1$ endplate).

\section{Statistical Analysis}

Analyses were performed using Statistical Package for the Social Sciences version 20.0 (SPSS Inc, Chicago, Illinois) and $\mathrm{R}$ Statistical Package. ${ }^{16}$ Variables were assessed for normality utilizing the Shapiro-Wilks test. Independent $t$ tests, MannWhitney $U$, and $\chi^{2}$ comparisons were used to compare continuous (patient demographics, radiographic measurements, and HRQoL data) and categorical variables (gender, comorbidities) between "new-onset CD" versus "Jmaintained CA" patient groups at each follow-up time interval. Variables with $P$ values of $<.1$ on univariate analyses were considered potential predictors of new-onset $\mathrm{CD}$. These were evaluated in multivariable binary logistic regression models built with backward elimination to determine independent predictors of developing new-onset $\mathrm{CD}$ at each follow-up interval. Paired $t$ tests and Wilcoxon signed-rank tests were utilized to compare changes in cervical and thoracolumbar sagittal alignment from baseline to subsequent yearly follow-up intervals. Multivariate repeated-measures mixed models measured the impact of new-onset $\mathrm{CD}$ on HRQoL scores at 1- and 2-year follow-up visits. The models were adjusted for known confounders of HRQoL, including age and baseline comorbidity status (Charlson Comorbidity Index score). A $P$ value of $<.05$ was used for statistical significance, and odds ratios are reported as (OR $[95 \% \mathrm{CI}], P$ value). Parametric and nonparametric tests were utilized appropriately.

\section{RESULTS}

\section{Patient Population and Cervical Radiographic Profile}

A total of 143 patients met inclusion criteria, amongst which 123 were female $(86.0 \%)$. The overall cohort had a mean age of $54.0 \pm 15.3$ years (range: $18-81$ years), and an average body mass index of $25.6 \pm 6.1 \mathrm{~kg} / \mathrm{m}^{2}$ (range: $17.0-48.3 \mathrm{~kg} / \mathrm{m}^{2}$ ). At baseline, $88(61.5 \%)$ patients were classified as showing CA while 55 (38.5\%) had CD based on the respective specified criteria for each group. Regarding deformity thresholds, $51.7 \%$ of patients met the CK threshold $\left(>10^{\circ}\right), 58.7 \%$ met $\mathrm{T} 1 \mathrm{~S}-\mathrm{CL}$ threshold $\left(>20^{\circ}\right)$, and $42.0 \%$ met SVA threshold $(>40 \mathrm{~mm})$. Table 1 presents baseline demographic and radiographic profiles of the CA groups compared to the remaining patients. The CA group at 
Table 1. Univariate (independent $t$ test and $\chi^{2}$ ) results for comparison of baseline patient demographic characteristics and radiographic measurements between cervically aligned patients at baseline and the remaining study cohort.

\begin{tabular}{|c|c|c|c|}
\hline Baseline Variables & Cervical Alignment $(\mathbf{n}=\mathbf{8 8})$ & Remaining Cohort $(\mathrm{n}=\mathbf{8 2})$ & $P$ \\
\hline \multicolumn{4}{|l|}{ Patient Demographics } \\
\hline Age, y & $52.68 \pm 15.11$ & $54.94 \pm 15.38$ & .384 \\
\hline Weight, kg & $66.49 \pm 13.71$ & $71.66 \pm 21.49$ & .085 \\
\hline BMI, $\mathrm{kg} / \mathrm{m}^{2}$ & $24.56 \pm 4.47$ & $26.40 \pm 6.94$ & .059 \\
\hline$\%$ Female & $85.0 \%$ & $86.7 \%$ & .475 \\
\hline \multicolumn{4}{|l|}{ Comorbidities } \\
\hline Prior spine surgery, \% & $15.3 \%$ & $19.8 \%$ & .324 \\
\hline Arthritis, \% & $18.3 \%$ & $22.9 \%$ & .328 \\
\hline Depression, \% & $16.7 \%$ & $9.8 \%$ & .160 \\
\hline Diabetes, $\%$ & $3.3 \%$ & $3.6 \%$ & .650 \\
\hline Hypertension, \% & $10.0 \%$ & $25.3 \%$ & $.016^{*}$ \\
\hline Neurologic, $\%$ & $3.3 \%$ & $1.2 \%$ & .380 \\
\hline Osteoporosis, \% & $8.3 \%$ & $10.8 \%$ & .421 \\
\hline Smoking history, \% & $11.7 \%$ & $8.5 \%$ & .366 \\
\hline \multicolumn{4}{|l|}{ Radiographic parameters } \\
\hline Baseline PT & $18.35 \pm 9.28$ & $22.08 \pm 10.08$ & $.025 *$ \\
\hline Baseline PI & $52.84 \pm 11.46$ & $57.52 \pm 13.08$ & $.028 *$ \\
\hline Baseline SS & $34.49 \pm 10.41$ & $35.46 \pm 10.63$ & .589 \\
\hline Baseline PI - LL & $3.32 \pm 14.48$ & $8.48 \pm 16.81$ & .059 \\
\hline Baseline T1S - CL & $12.43 \pm 5.36$ & $25.24 \pm 5.02$ & $<.001 *$ \\
\hline Baseline C2-C7 CL & $13.33 \pm 12.14$ & $-3.54 \pm 11.84$ & $<.001 *$ \\
\hline Baseline C2-C7 SVA & $28.42 \pm 17.20$ & $29.59 \pm 14.07$ & .719 \\
\hline Baseline $\mathrm{C} 2-\mathrm{T} 3$ & $9.97 \pm 13.07$ & $-3.54 \pm 11.84$ & $<.001 \%$ \\
\hline Baseline C2 slope & $11.34 \pm 5.54$ & $23.10 \pm 5.39$ & $<.001 *$ \\
\hline
\end{tabular}

Abbreviations: BMI indicates body mass index; PT, pelvic tilt; PI, pelvic incidence; SS, sacral slope; PI - LL, mismatch between pelvic incidence and lumbar lordosis; T1S - CL, mismatch between T1 slope and cervical lordosis; CL, cervical lordosis; and SVA, sagittal vertical axis.

*Bolded cells are statistically significant to $P<.05$.

baseline had mean age of $52.7 \pm 15.1$ years, mean body mass index of $24.6 \pm 4.5 \mathrm{~kg} / \mathrm{m}^{2}$, and comprised $85.0 \%$ females. Cervical alignment patients, as demonstrated by their radiographic profile categorization, displayed smaller T1S - CL (12.4 versus 5.4, $P<.001$ ) and larger $\mathrm{C} 2-\mathrm{C} 7$ lordosis (13.3 versus $-3.4, P=.002$ ).

\section{New-Onset CD}

The prevalence of new-onset $\mathrm{CD}$ from baseline CA patients was $30.0 \%$ and $41.7 \%$ at 1 - and 2 -year follow-up examinations, respectively (Figure 2). In

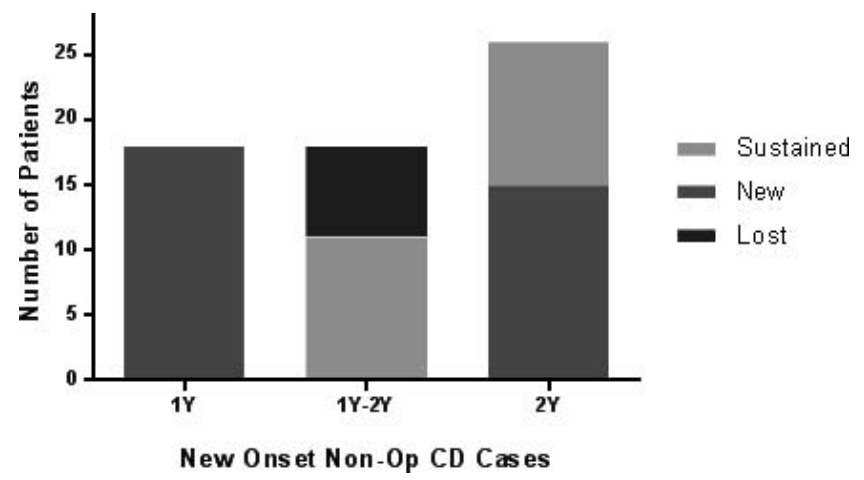

Figure 2. Distribution of new onset nonoperative cervical deformity cases at baseline and at 1 and 2 years. CD indicates cervical deformity; new, unique patients displaying new onset $C D$ at 1 year $(n=18)$; sustained, patients with new-onset $C D$ that persisted from 1 year to 2 years $(n=11)$; lost, patients whose new-onset CD corrected from 1-year to 2-year follow-up $(n=7)$. all, 18 patients with $\mathrm{CD}$ at the 1-year follow-up were de novo cases. Out of these, 11 patients $(18.3 \%)$ maintained this new-onset CD from 1 to 2 years, whereas 7 patients $(11.7 \%)$ improved their CA at the 2-year follow-up, mainly due to spontaneous restoration of normal C2-C7 SVA $(n=6)$. At the 2year follow-up, $14(23.3 \%)$ new patients developed CD. Following initial CA evaluation within the total cohort, the trend for $\mathrm{C} 2-\mathrm{C} 7 \mathrm{CK}$ as the most common presentation of CD persisted among newonset CD patients at each follow-up time point (Table 2): C2-C7 cervical lordosis rates at 1- and 2year follow-up visits were $56.7 \%$ and $55.0 \%$.

Table 2. Comparison of thoracolumbar sagittal profiles changes (gauged by pelvic tilt, thoracic kyphosis, and global angulation) at each follow-up interval for adult spinal deformity patients that were cervically aligned and deformed at baseline.

\begin{tabular}{lccc}
\hline $\begin{array}{l}\text { Sagittal Parameter } \\
\text { (Difference From } \\
\text { Baseline to X Year) }\end{array}$ & $\begin{array}{c}\text { Cervical } \\
\text { Alignment }\end{array}$ & $\begin{array}{c}\text { Cervical } \\
\text { Deformity }\end{array}$ & $\boldsymbol{P}$ \\
\hline 1-year follow-up & & & \\
S1 PT (PT) & $0.39(4.10)$ & $-0.76(3.16)$ & .410 \\
T4-T12 (TK) & $2.96(6.60)$ & $2.80(5.72)$ & .945 \\
C2-S1 (GA) & $1.11(7.90)$ & $-3.82(5.34)$ & .090 \\
2-year follow-up & & & \\
S1 PT (PT) & $0.212(2.80)$ & $1.78(5.43)$ & .277 \\
T4-T12 (TK) & $-0.83(5.62)$ & $-1.48(8.80)$ & .801 \\
C2-S1 (GA) & $-1.40(6.43)$ & $-8.11(13.46)$ & .069 \\
\hline
\end{tabular}

Abbreviations: PT indicates pelvic tilt; TK, thoracic kyphosis; and GA, global angulation. 
Table 3. Univariate (independent $t$ tests, and Mann-Whitney $U$ and $\chi^{2}$ analyses) results for the comparison of baseline sagittal parameters of new-onset cervical deformity patients to those with maintained cervical alignment at 1 - and 2 -year follow-up examinations. All included variables with $P<.1$ were assessed in multivariate models.

\begin{tabular}{|c|c|c|c|}
\hline & Maintained CA & New-Onset CD & $P$ \\
\hline \multicolumn{4}{|l|}{1 year postenrollment } \\
\hline Baseline weight & $64.17 \pm 12.67$ & $71.77 \pm 14.88$ & $.049 *$ \\
\hline Diabetes & $0.0 \%$ & $11.1 \%$ & .086 \\
\hline Prior history of spine surgery & $7.3 \%$ & $33.3 \%$ & $.018 *$ \\
\hline Baseline T1 slope & $22.76 \pm 8.57$ & $29.39 \pm 12.84$ & $.022 *$ \\
\hline Baseline C2-C7 CL & $10.41 \pm 10.26$ & $16.80 \pm 15.03$ & .061 \\
\hline Baseline C2-C7 SVA & $23.48 \pm 14.75$ & $39.93 \pm 17.38$ & $<.001 \%$ \\
\hline Baseline C2-T3 SVA & $51.00 \pm 13.73$ & $66.96 \pm 30.93$ & $.007 *$ \\
\hline Baseline C2-S1 SVA & $29.14 \pm 41.42$ & $51.23 \pm 46.56$ & .073 \\
\hline \multicolumn{4}{|l|}{2 years postenrollment } \\
\hline Baseline C2-C7 SVA & $23.22 \pm 14.23$ & $35.70 \pm 18.60$ & $.005 *$ \\
\hline Baseline C2 slope & $10.05 \pm 5.83$ & $13.14 \pm 4.64$ & $.032 *$ \\
\hline
\end{tabular}

Abbreviations: CA indicates cervical alignment; CD, cervical deformity; CL, cervical lordosis; and SVA, sagittal vertical axis.

*Bolded cells are statistically significant to $P<.05$.

Significant differences in baseline demographic and radiographic parameters in new-onset $\mathrm{CD}$ and maintained CA groups are reported in Table 3. Patients with 1-year new-onset CD included fewer females (CD: $66.7 \%$ versus CA: $92.9 \% ; P=.016)$ and had greater weight (CD: 71.8 versus CA: $64.2 \mathrm{~kg}$; $P=.049)$ compared to maintained CA patients. The 2 groups differed predominantly on the basis of baseline radiographic parameters at each follow-up interval: baseline C2-C7 SVA was significantly larger in newonset CD nonoperative patients at 1 year (CD: 39.9 versus CA: $23.5 \mathrm{~mm} ; P<.001)$, and 2 years (CD: 34.8 versus CA: $23.5 \mathrm{~mm} ; P=.010)$; baseline C2-T3 SVA was significantly larger in new-onset $\mathrm{CD}$ patients at 1 year (CD: 67.0 versus CA: $51.0 \mathrm{~mm}$; $P<.001$ ); newonset $\mathrm{CD}$ patients displayed larger $\mathrm{T} 1$ slope at 1-year follow-up (CD: $29.4^{\circ}$ versus CA: $22.8^{\circ} ; P=.022$ ), and greater $\mathrm{C} 2$ slope at 2 years $\left(\mathrm{CD}: 13.3^{\circ}\right.$ versus $\mathrm{CA}: 9.8^{\circ}$; $P=.016$ ). Additionally, patients that developed de novo CD at 2 years displayed significant deterioration of alignment incorporating the cervicothoracic junction: T1 slope, C2-T3 angle, and C2-T3 positive translation all significantly increased during nonoperative treatment $(P<.023$ all cases $)$ (Table 4$)$.

Table 4. Paired $t$ tests and Wilcoxon signed-rank tests for cervical and spino-pelvic parameter changes from baseline to 1- and 2-year follow-up, for CD-1Y and CD$2 Y$ deformity groups, respectively. Numbers in parentheses are standard deviations.

\begin{tabular}{|c|c|c|c|c|c|c|}
\hline \multirow[b]{3}{*}{ Parameters } & \multicolumn{6}{|c|}{ New-Onset Cervical Deformity Group } \\
\hline & \multicolumn{3}{|c|}{ CD-1Y } & \multicolumn{3}{|c|}{ CD-2Y } \\
\hline & Baseline & & 1 Year & Baseline & & 2 Years \\
\hline cSVA, mm & $45.00 \pm 15.88$ & 0.341 & $48.04 \pm 9.53$ & $35.10 \pm 19.98$ & 0.674 & $36.58 \pm 17.69$ \\
\hline $\mathrm{CL}, \stackrel{\circ}{\circ}$ & $19.25 \pm 13.90$ & 0.115 & $22.17 \pm 11.99$ & $5.70 \pm 18.73$ & 0.081 & $10.33 \pm 13.73$ \\
\hline C2-T3 SVA, mm & $78.22 \pm 21.85$ & 0.117 & $84.74 \pm 17.03$ & $59.76 \pm 31.88$ & $0.014 *$ & $66.34 \pm 27.93$ \\
\hline $\mathrm{C} 2-\mathrm{T} 3,^{\circ}$ & $10.25 \pm 15.37$ & $0.013 *$ & $16.95 \pm 14.11$ & $-0.26 \pm 16.25$ & $0.004 *$ & $7.51 \pm 14.25$ \\
\hline T1 Slope, ${ }^{\circ}$ & $29.39 \pm 12.84$ & 0.368 & $30.74 \pm 13.48$ & $25.06 \pm 12.57$ & $0.023 *$ & $28.58 \pm 13.44$ \\
\hline $\mathrm{SS}, \stackrel{\circ}{2}$ & $32.98 \pm 8.45$ & 0.129 & $34.31 \pm 9.58$ & $34.64 \pm 7.34$ & 0.286 & $35.56 \pm 8.93$ \\
\hline $\mathrm{PT}, \stackrel{\circ}{\circ}$ & $19.11 \pm 8.44$ & 0.177 & $17.72 \pm 7.20$ & $19.27 \pm 8.59$ & 0.701 & $19.59 \pm 9.86$ \\
\hline $\mathrm{PI}-\mathrm{LL},{ }^{\circ}$ & $4.88 \pm 12.74$ & 0.829 & $5.18 \pm 14.07$ & $3.88 \pm 12.54$ & 0.331 & $5.24 \pm 14.96$ \\
\hline $\mathrm{SVA}, \mathrm{mm}$ & $24.16 \pm 44.68$ & 0.159 & $33.52 \pm 43.43$ & $14.66 \pm 50.03$ & 0.314 & $21.15 \pm 38.75$ \\
\hline $\mathrm{TK},{ }^{\circ}$ & $36.06 \pm 19.67$ & 0.383 & $34.74 \pm 18.66$ & $34.48 \pm 16.53$ & 0.964 & $34.56 \pm 16.72$ \\
\hline LL, ${ }^{\circ}$ & $47.23 \pm 11.06$ & 0.768 & $46.86 \pm 13.02$ & $50.03 \pm 11.11$ & 0.728 & $49.54 \pm 14.36$ \\
\hline $\mathrm{C} 2-\mathrm{S} 1, \circ$ & $22.05 \pm 10.94$ & 0.078 & $17.99 \pm 12.88$ & $14.35 \pm 10.61$ & $0.001 *$ & $22.50 \pm 8.06$ \\
\hline
\end{tabular}


Table 5. Binary logistic regression modeling for potential independent predictors of new-onset nonoperative cervical deformity at each follow-up year based on significant factors derived from previous univariate analysis.

\begin{tabular}{llll}
\hline & $\begin{array}{c}\text { Odds } \\
\text { Ratio }\end{array}$ & $\begin{array}{c}\mathbf{9 5 \%} \text { CI } \\
\text { Lower-Upper) }\end{array}$ & $\boldsymbol{P}$ \\
\hline 1-year follow-up & & & \\
Baseline weight, per 1-kg increase & 1.04 & $0.99-1.09$ & .137 \\
Prior history of spine surgery & 6.75 & $1.03-44.2$ & $\mathbf{. 0 4 7}$ \\
Baseline T1 slope, per 1-degree increase & 1.05 & $0.93-1.18$ & .436 \\
Baseline C2-C7 SVA, per 1-mm increase & 1.41 & $1.02-1.40$ & $\mathbf{. 0 2 5}$ \\
Baseline C2-T3 SVA, per 1-mm increase & 0.99 & $0.91-1.07$ & .726 \\
2-year follow-up & & & \\
Baseline C2 slope, per 1-degree increase & 1.12 & $1.00-1.24$ & $\mathbf{. 0 4 1 *}$ \\
Baseline C2-C7 SVA, per 1-mm increase & 1.04 & $1.00-1.08$ & $\mathbf{. 0 3 2 *}$ \\
\hline
\end{tabular}

Abbreviations: CI indicates confidence interval; SVA, sagittal vertical axis.

\section{Predicting New-Onset CD}

Positive predictors (those associated with higher odds of developing new onset CD) identified through multivariate analysis are reported in Table 5. Baseline C2-C7 SVA was significantly associated with increased risk of new-onset CD at 1-year (1.14 [1.02-1.40]; $P=.025)$ and 2-year (1.04 [1.00-1.08]; $P=.032)$ follow-up. A prior history of spine surgery at baseline was associated with new-onset $\mathrm{CD}$ risk at 1 year (6.75 [1.029-44.23]; $P=.047)$. Baseline C2 slope was also associated with increased risk of $\mathrm{CD}$ at 2-year follow-up (1.12 [1.00-1.24]; $P=.041)$.

\section{Sagittal Profile Comparison}

Patients displaying new-onset $\mathrm{CD}$ at 1- and 2year follow-up were evaluated for changes in their global sagittal spino-pelvic profiles (measured with thoracic kyphosis, global angulation, SVA, T1 pelvic angle) in comparison to patients that maintained CA. Both groups were consistently statistically similar from baseline to each follow-up period $(P>.05$ for all cases each year; Table 6$)$.

Table 6. Comparison of thoracolumbar sagittal profiles changes at each follow-up interval (1 and 2 years) for adult spinal deformity patients that were cervically aligned and developed new-onset deformity at 1- and 2-year followup.

\begin{tabular}{|c|c|c|c|}
\hline $\begin{array}{l}\text { Sagittal Parameter } \\
\text { (Difference From } \\
\text { Baseline to X year) }\end{array}$ & $\begin{array}{c}\text { New-Onset } \\
\text { Cervical Deformity }\end{array}$ & $\begin{array}{c}\text { Maintained } \\
\text { Cervical Alignment }\end{array}$ & $P$ \\
\hline \multicolumn{4}{|l|}{ 1-year follow-up } \\
\hline C7-S1 SVA, mm & $9.38 \pm 26.98$ & $4.32 \pm 22.79$ & .459 \\
\hline T4-T12 TK, ${ }^{\circ}$ & $1.32 \pm 6.24$ & $2.14 \pm 7.68$ & .691 \\
\hline TPA, ${ }^{\circ}$ & $0.91 \pm 2.63$ & $0.31 \pm 2.16$ & .359 \\
\hline $\mathrm{C} 2-\mathrm{S} 1 \mathrm{GA},{ }^{\circ}$ & $-4.06 \pm 7.92$ & $-0.10 \pm 7.40$ & .095 \\
\hline \multicolumn{4}{|l|}{ 2-year follow-up } \\
\hline C7-S1 SVA, mm & $6.48 \pm 31.59$ & $3.48 \pm 20.33$ & .658 \\
\hline T4-T12 TK, ${ }^{\circ}$ & $-0.80 \pm 8.80$ & $-0.16 \pm 7.96$ & .970 \\
\hline TPA, ${ }^{\circ}$ & $0.34 \pm 3.19$ & $0.24 \pm 2.08$ & .882 \\
\hline $\mathrm{C} 2-\mathrm{S} 1 \mathrm{GA},{ }^{\circ}$ & $-8.15 \pm 9.75$ & $-1.68 \pm 6.74$ & .200 \\
\hline
\end{tabular}

Abbreviations: SVA indicates sagittal vertical axis; TK, thoracic kyphosis; TPA, T1 pelvic angle; GA, global angulation.

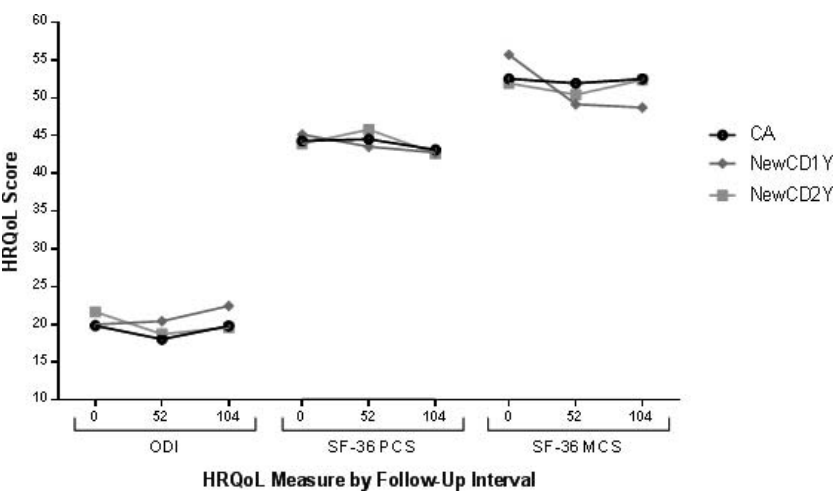

Figure 3. Patient-reported health-related quality of life at baseline, 1-year follow-up, and 2-year follow-up for Oswestry Disability Index (ODI), and Short Form 36 (SF-36) physical and mental component scores for cervically aligned patients at baseline and those that developed new-onset cervical deformity at each year. Abbreviations: HRQoL, health-related quality of life; PCS, physical component score; MCS, mental component score; CA, cervically aligned; NewCD1Y, new-onset cervical deformity at 1 year; NewCD2Y, new-onset cervical deformity at 2 years.

\section{Patient-Reported Outcomes}

Patient-reported outcomes are displayed in Figure 3. For nonoperative patients that developed new-onset $\mathrm{CD}$ at 1-year follow-up, there were no differences in HRQoL scores compared to CA patients in all categories $(P>.05)$. By 2-year follow-up, only the SRS mental score difference from baseline to 2 years was significantly greater in new-onset $C D$ patients (new-onset CD: 4.34 versus CA: $0.11 ; P=.018)$. Multivariate measures mixed models revealed that at 1 - and 2-year follow-up for nonoperative patients, development of $\mathrm{CD}$ did not significantly impact patient-reported outcomes, including SRS satisfaction, in all considered categories $(P>.05)$.

\section{DISCUSSION}

In contrast to thoracolumbar malalignment, there is a relative paucity of literature characterizing CDs, thereby leading to wide variations in surgical planning and management of these conditions among spine surgeons. The cervical spine's structural complexity and functional associations with lower spinal regions make it a critical area for alignment studies however, and many patients may exhibit simultaneous ASD and CD (CD). The optimal treatment algorithm, whether conservative or operative, and clinical importance of these pathologies, however, is not yet known, especially for cases in which CD develops secondary to ASD. This study is one of the first to consider this, by implementing a comprehensive, 3-part definition for 
$\mathrm{CA}$ and $\mathrm{CD}$ based on recently published literature to evaluate de novo $\mathrm{CD}$ for ASD patients treated nonoperatively at an extended follow-up. ${ }^{7}$

The baseline rate of CA was $61.5 \%$, with subsequent development of CD observed in $30.0 \%$ of patients at 1 -year and $41.7 \%$ at 2-year follow-up, for an average rate of $35.9 \%$. Patients were classified with $\mathrm{CD}$ based on criteria suggested by Passias et al: C2-C7 SVA $>4 \mathrm{~cm}, \mathrm{~T} 1 \mathrm{~S}-\mathrm{CL}>20^{\circ}$, and $\mathrm{C} 2-\mathrm{C} 7 \mathrm{Cobb}$ angle $>10^{\circ} .{ }^{7}$ While not otherwise investigated in a nonoperative setting, Passias et al implemented this radiographic definition to evaluate new-onset CD following ASD surgical correction, reporting a rate of $47.7 \%$. This rate is similar to the present study's, signifying that the natural history of CD development in ASD patients remains unaltered by surgical intervention. The increase in new-onset CD prevalence from 1-2 years observed in this study may reflect well-known reciprocal changes and mechanisms in operative ASD patients with cervical pathology as a means of restoring and maintaining horizontal gaze. ${ }^{12}$ Our results reflect how compensation occurs initially with ASD onset, but that CD progression may develop independently and in a nonoperative ASD population, CD is not solely attributable to surgical overcorrection and postoperative disease progression. We also observed that C2-C7 CK $<-10^{\circ}$ was the most prevalence driver of $\mathrm{CD}$ at each follow-up year. This is consistent with prior reports, such as that of $\mathrm{Yu}$ et al, noting that CK characterized cervical malalignment in $40 \%$ of cases. ${ }^{17}$ The high concurrence of ASD with CD in nonoperative and operative settings reveals that the cervical spine's self-corrective capacity is more limited in ASD cases as anticipated, and that ASD itself is partially responsible for the CA changes that are occurring; the importance of future study lies then in the efficacy and type of treatment for these cases.

New-onset CD has not been clinically evaluated in nonoperative patients. Despite elevated deformity rates, de novo $\mathrm{CD}$ occurrence at each follow-up year largely did not impact patient outcomes, determined by the Oswestry Disability Index, SF-36, and SRS$22 \mathrm{r}$ questionnaires. The rates of change and overall change were also similar for the yearly new-onset $\mathrm{CD}$ groups compared to CA patients, with the exception of SRS mental baseline-to-2-year difference, which was higher $\mathrm{CD}$ cases, indicating less improvement. These results may point to progressive neurological deficits linked with $\mathrm{CD}$, and it is possible that our observation of progressive $C D$ in nonoperative patients may result in neurological impairment, consistent with the natural history of several cervical pathologies left untreated. ${ }^{18,19}$ Effectively, Grosso et al found a significant relationship between modified Japanese Orthopaedic Association (mJOA) improvement, achievement of postoperative cervical lordosis, and greater degree of focal kyphosis correction in the setting of CD corrective surgery. ${ }^{20}$ Nonetheless, our results pertain to the uncertain position $\mathrm{CD}$ retains, when present in conjunction with ASD, on patient outcomes which was also initially highlighted recently by Passias et al. ${ }^{7}$

First-visit identification of those ASD patients at risk for new $\mathrm{CD}$ is crucial and can consequently alter a surgeon's plan of treatment. Increased baseline C2-C7 SVA and C2 slope were preoperative radiographic markers associated with increased odds of developing new onset $\mathrm{CD}$ after conservative ASD treatment at both 1 and 2 years. These measures likely reflect overall balance of the head over the spine. Particularly with respect to $\mathrm{C} 2-\mathrm{C} 7$ SVA, it is not surprising that greater baseline deformity links patients to subsequent upper spinal malalignment: Passias et al observed, for instance, that surgical ASD patients that remained malaligned 2 years postoperatively had larger preoperative C2-C7 SVAs $^{11}$; similarly, $\mathrm{Oh}$ et al noted significant deteriorative changes in C2-C7 SVA at 3 months and 2 years following thoracic deformity correction among 57 ASD patients. ${ }^{8}$ These authors also observed that among 22 ASD patients with preoperative C2-C7 SVA $>4 \mathrm{~cm}$, cervical malalignment persisted in $74 \%$ of cases, and was only corrected in $26 \%{ }^{8}$ Similarly, in an analysis of 470 adults with thoracolumbar deformities, Smith et al saw significantly greater cervical sagittal malalignment $(>4 \mathrm{~cm})$ in patients with higher C7-S1 SVA and pelvic tilt. ${ }^{6}$ These identified factors predisposing ASD patients to CD should be taken into consideration by health-care providers in planning and identifying at-risk patients.

Recent findings suggest that the ability of cervical spine to compensate for global sagittal malalignment can be reversible or permanent. For example, Smith et al demonstrated spontaneous improvement of cervical hyperlordosis following correction of thoracolumbar deformities. ${ }^{12}$ Conversely, Oh et al noted that the cervical malalignment corrected in only $26 \%$ of patients operated for thoracic defor- 
mity. ${ }^{8}$ In our study, we noted a progressive trend for increase in the rate of $\mathrm{CD}$ development with subsequent follow-up, despite no significant changes in global deformity parameters compared to maintained CA cases. The observation here that CD progression may develop independently in patients with ASD suggests that it cannot be solely attributable to compensatory mechanisms or surgical overcorrection. ${ }^{11,21,22}$ ASD treated conservatively may result in a reduced capacity for selfcorrection. ${ }^{11}$ New CD may then be a marker of potential disease progression, or more severe pathology despite similar global measurements. The finding that a prior history of spine surgery increased the risk of new-onset CD at 1-year followup in this study also underscores this, and retains clinical value in considering the protracted risk inherent in treating ASD. These identified factors predisposing ASD patients to $\mathrm{CD}$ are useful in guiding surgeons in treatment course planning and identifying at-risk patients.

Our results hinge on effectively defining $\mathrm{CD}$ and alignment, which remains difficult. The criteria in this report retain validity across other studies quantifying $C D$. Cervical sagittal spine plane translation is most commonly measured with C2C7 SVA, which is particularly relevant as it has been directly correlated with poorer SF-36 Physical Component Score and neck disability index outcomes. ${ }^{23}$ Multiple studies have defined a 4-cm C2C7 SVA threshold for deformity. ${ }^{7,23}$ Recent presented data from Protopsaltis et al. proposed $\mathrm{T} 1 \mathrm{~S}-\mathrm{CL}>20^{\circ}$ as effective in quantifying $\mathrm{CD},{ }^{24}$ and this cutoff has since been implemented in numerous CD studies, such as those by Passias et al and Lee et al. ${ }^{7,9}$ Clinical evaluations for CK remain to be fully proposed, but we built on use of $\mathrm{C} 2-\mathrm{C} 7 \mathrm{CK}>0^{\circ}$ by Smith et al as a measure of CD in our study. ${ }^{6}$ The most recent, and most widely accepted definitions for $\mathrm{CD}$, utilizing previously discussed sagittal radiographic regional and global parameters, are described in the Ames-International Spine Study Group CD classification. ${ }^{25}$ In applying the 3 measures of C2-C7 SVA, T1S - CL, and CK in unison, we aimed to target those patients with stricter CDs.

While typically presented as the first line of management, the utility of nonoperative ASD treatment is contested. Indeed, surgery for ASD is consistently well supported in the literature, but studies have been finding value in nonoperative treatment for ASD from a HRQoL standpoint. ${ }^{26-28}$ Liu et al noted that though operative ASD patient did demonstrate significant mean improvement and met minimal clinically important difference (MCID) thresholds, nonoperative patients still improved in at least 1 outcome measure at 1 -year follow-up. ${ }^{29}$ Specifically, nonoperative ASD patients significantly improved in SRS pain evaluation from baseline to 1 year, and more than 50\% gained MCID in 1 year Oswestry Disability Index, SRS pain, SRS appearance, and SRS mental scores. ${ }^{29}$ In one of the few studies looking at $\mathrm{CD}$ in nonoperative patients, Schwartz et al observed a worsening in CK $\left(>10^{\circ}\right.$, similar to our criteria for deformity) for pediatric posttraumatic patients receiving conservative treatment. ${ }^{30}$ The progression of CD noted in our present study parallels this study's results. The development of CD as secondary to ASD, as seen in this study, clearly demonstrates the need for further inquiry into the mechanistic progression of these spinal deformities, and how treatment can influence it.

\section{Limitations}

This study is limited in its retrospective design, and that the primary inclusion criterion for inclusion into the multicenter database was an ASD diagnosis. Further, current HRQoL measures are not specific to cervical pathology. Thoracolumbar deformity may also overwhelming influence currently utilized HRQoL metrics. A lack of HRQoL sensitivity for cervical pathology may limit its ability to accurately capture the clinical effects of $\mathrm{CD}$ development. Variations in radiographic protocol (patient positioning, especially) may also exist between enrollment sites, thereby potentially impacting radiographic determination of deformity and measurements. Incomplete short-term followup data was a limitation belonging to the multicenter database used as well. While all patients within this study had complete baseline, 1-year, and 2-year follow-up data, investigation of shorter follow-up time points $(<1$ year $)$ are needed for a more complete assessment of CA changes amongst nonoperative ASD patients. Additionally, though our implemented definitions for CD and CA have been previously used in the literature, these criteria still warrant further study and refinement. Lastly, a large proportion of included patients in this study were female, thereby potentially limiting the generalizability of these results. 


\section{Conclusion}

This study is the first assessing cervical radiographic changes concomitant with established thoracolumbar sagittal deformities in nonoperative patients at extended follow-up. Of 143 nonoperative ASD patients, $61.5 \%$ of patients were classified as cervically aligned at baseline; subsequent de novo regional deformities occurred at 1- and 2-year intervals, with a peak at 2 years $(41.7 \%)$. The most consistent form of new-onset CD was CK at each year. Progressive CDs manifested independently of thoracolumbar profile changes. Increased baseline C2-C7 SVA, C2 slope, and a prior surgical history increased new-onset $\mathrm{CD}$ odds at 1 and 2 years. These findings underscore the importance of full radiographic assessment and screening for CD at first visit among ASD patients for those individuals at risk for developing $\mathrm{CD}$, particularly in cases seeking nonoperative care to optimize prolonged treatment.

\section{REFERENCES}

1. Klineberg E, Schwab FJ, Smith JS, Gupta MC, Lafage V, Bess S. Sagittal spinal pelvic alignment. Neurosurg Clin $N$ Am. 2013;24(2):157-162.

2. Ames CP, Smith JS, Scheer JK, et al. Impact of spinopelvic alignment on decision making in deformity surgery in adults: a review. J Neurosurg Spine. 2012;16(6):547-564.

3. Glassman SD, Schwab FJ, Bridwell KH, Ondra SL, Berven S, Lenke LG. The selection of operative versus nonoperative treatment in patients with adult scoliosis. Spine (Phila Pa 1976). 2007;32(1):93-97.

4. Smith JS, Klineberg E, Schwab F, et al. Change in classification grade by the SRS-Schwab adult spinal deformity classification predicts impact on health-related quality of life measures: prospective analysis of operative and non-operative treatment. Spine (Phila Pa 1976). 2013;38:1663-1671.

5. Scheer JK, Ames CP, Deviren V. Assessment and treatment of cervical deformity. Neurosurg Clin $N \mathrm{Am}$. 2013;24(2):249-274.

6. Smith JS, Lafage V, Schwab FJ, et al. Prevalence and type of cervical deformity among 470 adults with throacolumbar deformity. Spine (Phila Pa 1976). 2014;39(17):1001-1009.

7. Passias PG, Soroceanu A, Smith J, et al. Postoperative cervical deformity in 215 thoracolumbar patients with adult spinal deformity: prevalence, risk factors, and impact on patient-reported outcome and satisfaction at 2-year follow-up. Spine (Phila Pa 1976). 2015;40(5):283-291.

8. Oh T, Scheer JK, Eastlack R, et al. Cervical compensatory alignment changes following correction of adult thoracic deformity: a multicenter experience in 57 patients with a 2year follow-up. J Neurosurg Spine. 2015;22(June):1-8.

9. Lee JS, Youn MS, Shin JK, Goh TS, Kang SS. Relationship between cervical sagittal alignment and quality of life in ankylosing spondylitis. Eur Spine J. 2015;24(6):11991203.

10. Katsuura A, Hukuda S, Saruhashi Y, Mori K. Kyphotic malalignment after anterior cervical fusion is one of the factors promoting the degenerative process in adjacent intervertebral levels. Eur Spine J. 2001;10(4):320-324.

11. Passias PG, Soroceanu A, Scheer J, et al. Magnitude of preoperative cervical lordotic compensation and C2-T3 angle are correlated to increased risk of post-operative sagittal spinal pelvic malalignment in adult thoracolumbar deformity patients at 2-year follow-up. Spine J. 2015;15(8):1756-1763.

12. Smith JS, Shaffrey CI, Lafage V, et al. Spontaneous improvement of cervical alignment after correction of global sagittal balance following pedicle subtraction osteotomy. $J$ Neurosurg Spine. 2012;17(4):300-307.

13. Champain S, Benchikh K, Nogier A, Mazel C, De Guise J, Skalli W. Validation of new clinical quantitative analysis software applicable in spine orthopaedic studies. Eur Spine J. 2006;15(6):982-991.

14. Rillardon L, Levassor N, Guigui P, et al. [Validation of a tool to measure pelvic and spinal parameters of sagittal balance]. Rev Chir Orthop Reparatrice Appar Mot. 2003;89(3): 218-227. http://www.ncbi.nlm.nih.gov/pubmed/12844045. Accessed September 26, 2014.

15. Gupta M, Henry JK, Schwab F, et al. Dedicated spine measurement software quantifies key spino-pelvic parameters more reliably than traditional picture archiving and communication systems tools. Spine (Phila Pa 1976). 2016;41(1):E22E27.

16. R Core Team, Team RC. R: a language and environment for statistical computing. 2012. http://www.r-project.org/. Accessed April 17, 2018.

17. Yu M, Silvestre C, Mouton T, Rachkidi R, Zeng L, Roussouly P. Analysis of the cervical spine sagittal alignment in young idiopathic scoliosis: a morphological classification of 120 cases. Eur Spine J. 2013;22(11):2372-2381.

18. Todd AG. Cervical spine: degenerative conditions. Curr Rev Musculoskelet Med. 2011;4(4):168-174.

19. Kadaňka Z, Bednařík J, Novotný O, Urbánek I, Dušek L. Cervical spondylotic myelopathy: conservative versus surgical treatment after 10 years. Eur Spine J. 2011;20(9):1533-1538.

20. Grosso MJ, Hwang R, Mroz T, Benzel E, Steinmetz MP. Relationship between degree of focal kyphosis correction and neurological outcomes for patients undergoing cervical deformity correction surgery. J Neurosurg Spine. 2013;18(6):537-544.

21. Blondel B, Schwab F, Bess S, et al. Posterior global malalignment after osteotomy for sagittal plane deformity: it happens and here is why. Spine (Phila Pa 1976). 2013;38(7):E394-E401.

22. Scheer JK, Tang J A, Smith JS, et al. Cervical spine alignment, sagittal deformity, and clinical implications. $J$ Neurosurg Spine. 2013;19(2):141-159.

23. Tang JA, Scheer JK, Smith JS, et al. The impact of standing regional cervical sagittal alignment on outcomes in posterior cervical fusion surgery. Neurosurgery. 2012;71(3):662669; discussion 669.

24. Protopsaltis TS, Terran J, Bronsard N, et al. T1 slope minus cervical lordosis (TS-CL), the cervical answer to PI-LL, defines cervical sagittal deformity in patients undergoing thoracolumbar osteotomy. In: Cervical Spine Research Society (CSRS) Annual Meeting; December 5-7; 2013.

25. Ames CP, Smith JS, Eastlack R, et al. Reliability assessment of a novel cervical spine deformity classification system. J Neurosurg Spine. 2015:1-11. 
26. Blondel B, Schwab FJ, Ungar B, et al. Impact of magnitude and percentage of global sagittal plane correction on health-related quality of life at 2-years follow-up. Neurosurgery. 2012;71(2):341-348; discussion 348.

27. Bridwell KH, Glassman S, Horton W, et al. Does treatment (nonoperative and operative) improve the two-year quality of life in patients with adult symptomatic lumbar scoliosis: a prospective multicenter evidence-based medicine study. Spine (Phila Pa 1976). 2009;34(20):2171-2178.

28. Smith JS, Shaffrey CI, Berven S, et al. Improvement of back pain with operative and nonoperative treatment in adults with scoliosis. Neurosurgery. 2009;65(1):86-94.

29. Liu S, Schwab F, Smith JS, et al. Likelihood of reaching minimal clinically important difference in adult spinal deformity: a comparison of operative and nonoperative treatment. Ochsner J. 2014;14(1):67-77.

30. Schwarz N, Genelin F, Schwarz AF. Post-traumatic cervical kyphosis in children cannot be prevented by nonoperative methods. Injury. 1994;25(3):173-175.

Disclosures and COI: All authors are a part of the International Spine Study Group Foundation, which receives funding from a company through which this study was conducted. Funding for the International Spine Study Group Foundation is from research grants from DePuy Spine and individual donations.

Corresponding Author: Peter G. Passias, MD, New York Spine Institute, New York University Medical Center-Hospital for Joint Diseases, 301 East 17th St, New York, NY 10003. Phone: (516) 357-8777; Fax: (516) 357-0087; Email: peter. passias@nyumc.org.

Published 21 December 2018

This manuscript is generously published free of charge by ISASS, the International Society for the Advancement of Spine Surgery. Copyright (c) 2018 ISASS. To see more or order reprints or permissions, see http://ijssurgery.com. 